\title{
BMJ Open Prognostic impact of moderate or severe mitral regurgitation (MR) irrespective of concomitant comorbidities: a retrospective matched cohort study
}

\author{
Roshan Prakash, ${ }^{1}$ Matthew Horsfall, ${ }^{1}$ Andrew Markwick, ${ }^{1}$ Marsus Pumar, ${ }^{1}$ \\ Leong Lee, ${ }^{1}$ Ajay Sinhal, ${ }^{1}$ Majo X Joseph, ${ }^{1}$ Derek P Chew ${ }^{1,2}$
}

To cite: Prakash $\mathrm{R}$, Horsfall M, Markwick A, et al. Prognostic impact of moderate or severe mitral regurgitation (MR) irrespective of concomitant comorbidities: a retrospective matched cohort study. BMJ Open 2014;4:e004984. doi:10.1136/bmjopen-2014004984

- Prepublication history for this paper is available online. To view these files please visit the journal online (http://dx.doi.org/10.1136/ bmjopen-2014-004984).

Received 2 February 2014 Revised 12 June 2014 Accepted 30 June 2014

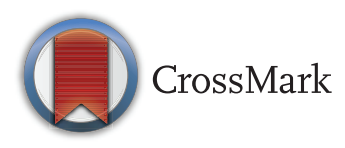

${ }^{1}$ Department of Cardiovascular Medicine, Flinders Medical Centre, Adelaide, South Australia, Australia

${ }^{2}$ Faculty of Medicine, Flinders University, Adelaide, South Australia, Australia

Correspondence to Dr Derek P Chew; derek.chew@flinders.edu.au

\section{ABSTRACT}

Objective: We sought to objectively quantify the independent impact of significant mitral regurgitation (MR) on prognosis in patients with multiple comorbidities and ascertain the extent to which median survival is affected by increasing comorbidities.

Methods: This was a retrospective matched cohort study using a clinical-echocardiography reporting database linked to a clinical and administrative database in an Australian tertiary hospital. We identified our study cohort (patients with significant MR) and control cohort (without MR) on transthoracic echocardiographies performed between 2005 and 2010. The main outcome measures were mortality and heart failure rehospitalisation. A Cox proportional hazards model was used to adjust for clinical covariates and the 'win ratio' methodology was utilised to estimate the impact of MR on main outcomes.

Results: A total of 218 matched patients with and without significant MR were followed-up for 1 year. Significant MR was associated with an adjusted HR for mortality of 1.83 ( $95 \% \mathrm{Cl} 1.28$ to $2.62, p<0.001)$. The win ratio for death and death or heart failure readmission was $0.57(95 \% \mathrm{Cl} 0.40$ to 0.78 , $\mathrm{p}=0.0002)$ and $0.53(95 \% \mathrm{Cl} 0.39$ to $0.71, \mathrm{p}<0.0001)$, respectively. Significant MR with left ventricular (LV) systolic dysfunction and age between 75 and 85 years were associated with a substantial reduction in median survival by 2.3 years. Significant MR with LV systolic dysfunction, age beyond 85 and advance comorbidities were associated with a lesser reduction in median survival by 0.2 years.

Conclusions: Significant MR in patients with multiple comorbidities leads to increase in death and heart failure rehospitalisation with reduced estimated median survival. However, its impact diminishes with increasing comorbidities.

\section{INTRODUCTION}

Significant mitral regurgitation (MR) is the most frequently diagnosed valvular disorder in the USA ${ }^{1}$ and the second most common

\section{Strengths and limitations of this study}

- This study has evaluated the impact of mitral regurgitation (MR) from data derived from a clinical and echocardiographic-linked database of real-world patients. The tertiary hospital setting in which this study was conducted provided a platform for which patients with MR and multiple comorbidities were evaluated at baseline and followed up for a year. A similar group of patients without significant MR were matched and followed-up in a similar manner for major clinical outcomes such as mortality and heart failure rehospitalisation.

- Prior studies have unequivocally demonstrated the adverse impact of untreated MR with increased mortality, morbidity and heart failure rehospitalisation. The present study, while concurring with these prior observations, further demonstrates that, with increasing non-cardiac comorbidities in patients, the independent impact of MR appears to diminish. MR in the context of advanced age left ventricular dysfunction and advanced comorbidities has a lesser relative impact on clinical outcomes since other medical factors may play a more significant role.

- The study concludes that the relevance of the MR needs to be evaluated within the context of the greater burden of comorbid disease as this will help define the appropriate clinical population that should be studied in future clinical trials for emerging MR corrective technologies.

- This study is retrospective and observational in nature and the differences observed in median survival between various patient groups were best estimates from robust modelling. 
Untreated significant MR carries a dismal outlook with high morbidity and excess mortality. ${ }^{3-6}$ Surgical correction of significant MR, particularly mitral valve repair, when feasible, remains the only treatment modality hitherto, to successfully provide improvement in symptoms and heart failure. ${ }^{7}$ This mode of treatment is supported by major guidelines that provide high-level recommendations based on various clinical and echocardiographic criteria. $^{78}$

With progressive improvement in the diagnostic capabilities, improvement in quantification ${ }^{9}$ and surgical treatment of MR, ${ }^{10}$ a major aspect of the assessment of significant MR, its independent impact within the context of concomitant comorbidities, remains under-investigated. ${ }^{10}$ Previous observational and prospective studies have unequivocally demonstrated the adverse impact of MR on outcomes, but these studies have routinely separated the impact of MR from that of reduced left ventricular (LV) function. ${ }^{2-4} 610$ Other studies have either excluded patients with excessively high surgical risk due to multiple comorbidities or provided widely variable estimates of its impact on clinical outcomes. ${ }^{6}{ }^{11-16}$ Consequently, objective assessment of the impact of significant MR, in the context of concomitant comorbidities, is difficult to ascertain.

An improved understanding of the impact of significant MR, especially in the context of the greater burden of comorbidities, is required to improve decision-making and patient management. This will ultimately assist clinicians in refining patient selection for the best-suited therapeutic modality in the treatment of significant MR.

This study seeks to quantify the impact of significant MR on clinical outcomes such as death and heart failure, after accounting and within the context of age and comorbidities in a real-world population of patients undergoing echocardiography.

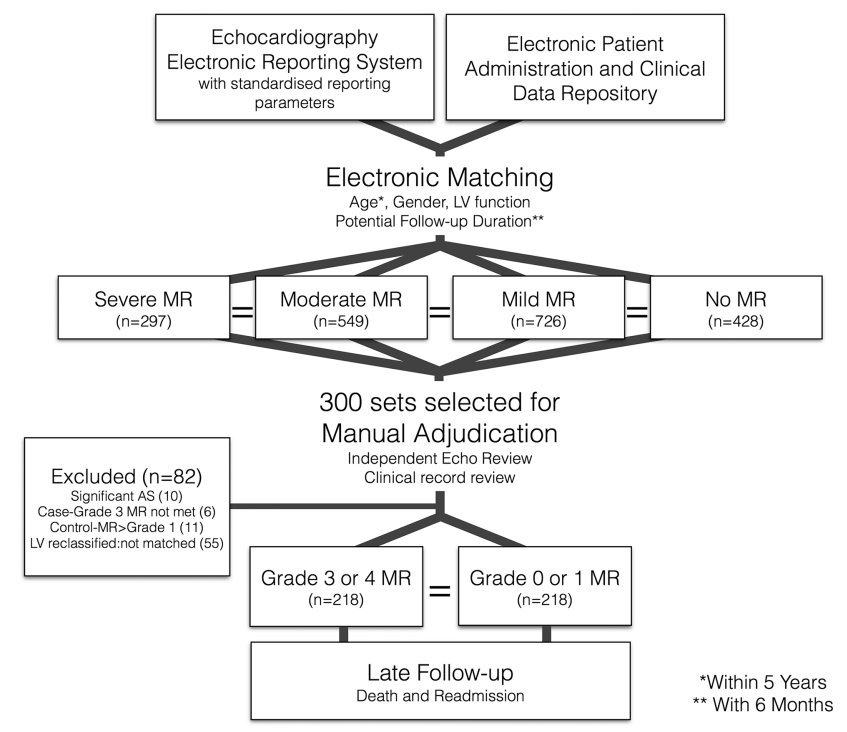

Figure 1 Study design: two-stage matching and adjudication process.

\section{METHODS}

Patients with significant MR were retrospectively identified from 2005 to 2010 through the Flinders Medical Centre Echocardiographic database. This clinical service provides regional echocardiographic imaging to a population of approximately 600000 and performs over 12000 echocardiograms per year.

\section{Electronic and echocardiographic matching process}

Patients were identified through a two-stage matching process (figure 1). Using an algorithm, patients with various degrees of reported MR (mild, moderate and severe) and no MR were matched by age (within 5 years), gender, left ventricular function ejection fraction (LVEF) (classified as <35\%, 35-44\%, 45-60\%, $>60 \%$ ) and date of echocardiogram (within 6 months) to permit comparable follow-up duration for those with and without MR. Patients with prior surgical repair or replacement of the mitral valve, moderate mitral stenosis and those with moderate or severe aortic stenosis were excluded. Through this method, 297 patients with severe MR were matched to 549 patients (1:1.9) with moderate MR, 726 patients (1:2.4) with mild MR and 428 patients (1:1.4) without MR.

\section{Matched population}

Subsequently, through independent adjudication by a trained echocardiography specialist, each echocardiogram was assessed. Patients with grade 3 or 4 MR (significant MR) were matched 1:1 to patients with either no or mild MR (without significant MR) by age (within 5 years), gender, LVEF classified as severe dysfunction $<35 \%$, moderate dysfunction 35-44\%, mild dysfunction $45-60 \%$, preserved $>60 \%$ and date of echocardiogram (within 6 months).

Recognising that LV function is a potent predictor of outcomes and the possibility of overestimating risk by matching this variable, an alternative-matching algorithm by NYHA class was performed. This algorithm did not reveal any significant differences when compared to the LV-based matching algorithm with respect to baseline characteristics, echocardiographic parameters and clinical outcomes. As such, the LV-based matching algorithm was utilised for this study and is presented.

All patients were eligible for more than the 12 month follow-up, enabling further clinical and echocardiographic evaluation. Clinical characteristics including, age, hypertension, diabetes, renal impairment, heart failure (New York Heart Association Class I-IV), liver disease, prior stroke, a history of atrial fibrillation, known ischaemic heart disease, prior coronary artery bypass grafting, prior lung disease and Charlson index were determined through electronic and medical record review. The Charlson index is a composite score incorporating multiple comorbidities and has been demonstrated to provide powerful prognostic information. ${ }^{17}$ 
Echocardiographic and clinical outcome variables

Two-dimensional transthoracic echocardiograms (2D-TTE) for patients with and without significant MR were reviewed and adjudicated by a cardiologist with level III training in TTE as defined by the American College of Cardiology/American Heart Association (ACC/AHA) Clinical Competence Statement on Echocardiography. ${ }^{18}$ When multiple echocardiograms were available, the first eligible echocardiogram was used. Each 2D-TTE study was independently reviewed and adjudicated by strict adherence to the American Society of Echocardiography (ASE) report on recommendations for evaluation of the severity of native valvular regurgitation with 2D and Doppler echocardiography. ${ }^{9}$ Echocardiographic parameters included quantitative parameters for MR; effective regurgitant orifice area (EROA) $\left(\mathrm{cm}^{2}\right)$, regurgitant fraction $(\%)$, regurgitant volume $(\mathrm{mL} /$ beat $)$, supportive signs of severity, vena contracta width, mitral inflow characteristics, colour flow jet area, pulmonary vein flow, pulmonary artery pressure and other volumetric parameters such as LVESD and LVEDD, left atrial area and LVEF; biplane Simpsons method. Aetiology of MR was classified as degenerative or functional according to the morphology of the mitral valve on 2D-TTE. Significant MR was considered to be present if any of the following echocardiographic features were present: vena contracta width greater than $0.7 \mathrm{~cm}$ with a large central MR jet ( $>40 \%$ of the left atrial area), regurgitant volume of $\geq 60 \mathrm{~mL} /$ beat, regurgitant fraction $\geq 50 \%$, EROA $\geq 0.4 \mathrm{~cm}^{2}$, systolic pulmonary vein flow reversal.

The date of the first echocardiogram meeting the criteria for MR or the matching control was used as the start (time zero) of this analysis. Subsequent clinical outcomes were determined through data-linkage using the South Australian Clinical Record Repository which records all primary, secondary and procedural information of all patients admitted to the South Australian public health system. Deaths were determined through linkage with the South Australian registry of births, deaths and marriages. While admissions for all diagnoses were available within this system, using ICD 10AM coding definitions, the analysis focused on admissions heart failure (I50), myocardial infarction (I21), atrial fibrillation (I48) and cerebrovascular disease (G46).

\section{Statistical analysis}

Baseline clinical and echocardiographic characteristics were compared between matched patients. Continuous variables were expressed as a mean $\pm \mathrm{SD}$ or median and IQR for variables with non-Gaussian distributions. All discrete variables were expressed as counts (n) and percentages $(\%)$ of the study population $(\mathrm{N})$. In order to assess the impact of severity of MR on mortality and recurrent hospitalisation for heart failure, a survival analysis was performed using both cohorts in an unadjusted manner and then adjusted using a Cox proportional hazards model including the following baseline clinical and echocardiographic variables: age, left ventricular function, New York Heart Association heart failure classification, anaemia, prior acute coronary syndrome (ACS) history of atrial fibrillation, prior coronary revascularisation, hypertension, dyslipidaemia, a history of diabetes, pulmonary hypertension, renal impairment (estimatedglomerular filtration rate by modification of diet in renal disease equation $<30 \mathrm{~mL} / \mathrm{min} / 1.73 \mathrm{~m}^{2}$ ), a history of malignancy and prior lung disease. Variables with a $\mathrm{p}$ value of $>0.2$ were removed from the model. Interactions between the aetiology of MR and outcome were sort but not found to be significant. Given the study design, a model was clustered on matched patients sets to allow for intragroup correlation hazards assumption was evaluated and found to be met. The utility of the final model was assessed visually by plotting the Cox Snell residuals against the Nelson-Aalen cumulative hazard. The fit of the model was found to be acceptable. The association between significant MR and freedom from death or heart failure hospitalisation in the entire cohort and among patients over the age of 75 years was plotted using Kaplan-Meier methods. To evaluate the time to first event of the composite of death or heart failure admission associated with significant MR, the 'win ratio' methodology was applied for a separate evaluation of the time to heart failure admission and death within each matched pair, before aggregating these estimates into a composite estimate. A ratio of $>1.0$ implies more 'wins' (ie, longer time to events), while a ratio of $<1.0$ implies more 'losses' (ie, shorter time to events) among the exposed patients. ${ }^{19} 20$

To estimate the impact of significant MR on median survival, the final Cox proportional hazards model was utilised to predict median survival times in both patient groups. To explore the impact of clinical comorbidities, median survival and relative and absolute reduction in survival attributable to MR, several clinical scenarios were modelled and displayed graphically. All analyses were undertaken using STATA MP V.13 (College Station, Texas, USA) and a p value of 0.05 was considered statistically significant.

\section{RESULTS}

\section{Baseline characteristics}

A total of 218 pairs of patients with and without significant MR were included in this analysis. Baseline characteristics are summarised in table 1 . The mean age of patients with significant MR was 78.9 years $( \pm 11.7)$, with $54.5 \%$ being female. Patients with significant MR were more likely to have a history of atrial fibrillation, an admission for heart failure and a history of coronary artery bypass grafting but were less likely to have experienced a prior cerebrovascular accident. The overall cohort was well matched for diabetes and hypertension but when LVEF was reclassified to label an EF $<60 \%$ as representing mild LV impairment, intragroup differences in LV classification emerged. Similarly, echocardiographic parameters among patients with significant 
Table 1 Demographic and clinical characteristics among patients with and without significant MR

\begin{tabular}{|c|c|c|c|}
\hline Characteristic & $\begin{array}{l}\text { Without significant MR } \\
\mathrm{N}=218\end{array}$ & $\begin{array}{l}\text { With significant } \\
\text { MR N=218 }\end{array}$ & p Value \\
\hline Age (years, SD) & $79.0(11.7)$ & $78.9(11.7)$ & 0.770 \\
\hline Female gender (n, \%) & $119(54.5)$ & $119(54.5)$ & 1.00 \\
\hline Diabetes (n, \%) & $50(22.9)$ & $51(23.4)$ & 0.910 \\
\hline Hypertension (n, \%) & $155(71.1)$ & $146(67.0)$ & 0.351 \\
\hline Prior ischaemic heart disease (n, \%) & 116 (53.2) & $113(51.8)$ & 0.774 \\
\hline Prior myocardial infarction & $109(50.0)$ & $103(47.2)$ & 0.565 \\
\hline Prior heart failure $(n, \%)$ & $43(19.7)$ & $63(28.9)$ & 0.026 \\
\hline NYHA class I & $136(62.4)$ & $122(55.9)$ & 0.113 \\
\hline NYHA class II & $20(9.2)$ & $14(6.4)$ & \\
\hline NYHA class III & $19(8.7)$ & $18(8.3)$ & \\
\hline NYHA class IV & $43(19.7)$ & $64(29.4)$ & \\
\hline Prior CVA (n, \%) & $62(28.4)$ & $43(19.7)$ & 0.033 \\
\hline Prior or known malignancy (n, \%) & $55(25.2)$ & $57(26.1)$ & 0.826 \\
\hline Prior atrial fibrillation $(\mathrm{n}, \%)$ & $79(36.2)$ & $121(55.5)$ & $<0.001$ \\
\hline Prior CABG & $21(9.6)$ & $42(29.3)$ & 0.004 \\
\hline Chronic airways disease (n, \%) & $53(24.3)$ & 39 (17.9) & 0.100 \\
\hline Peripheral vascular disease $(n, \%)$ & $30(13.8)$ & $35(16.1)$ & 0.501 \\
\hline GFR MDRD, $<30 \mathrm{~mL} / \mathrm{min} / 1.73 \mathrm{~m}^{2}(\mathrm{n}, \%)$ & $24(11.0)$ & $37(17.0)$ & 0.073 \\
\hline Charlson index (median, IQR) & $1(2)$ & $2(1)$ & 0.125 \\
\hline LVEF (mean, SD) & $49.9(18.1)$ & $49.9(18.5)$ & 0.909 \\
\hline \multicolumn{4}{|l|}{ LV class } \\
\hline Normal (n, \%) & $101(46.3)$ & $90(41.3)$ & 0.006 \\
\hline Mild (n, \%) & $21(9.6)$ & $44(20.2)$ & \\
\hline Moderate (n, \%) & $57(26.2)$ & 39 (17.9) & \\
\hline Severe $(n, \%)$ & 39 (17.9) & $45(20.6)$ & \\
\hline Systolic pulmonary artery pressure (median, IQR) & $20(31)$ & $28(21)$ & $<0.001$ \\
\hline LV systolic diameter (mm, mean, (SD)) & $35.9(1.3)$ & $38.9(2.1)$ & 0.027 \\
\hline LV diastolic diameter (mm, mean, (SD)) & $47.9(0.9)$ & $53.8(3.3)$ & 0.002 \\
\hline Left atrial area $\left(\mathrm{mm}^{2}\right.$, mean, $\left.(\mathrm{SD})\right)$ & $22.1(6.8)$ & $28.8(8.2)$ & 0.001 \\
\hline
\end{tabular}

MR demonstrated larger overall left heart chamber dimensions for LVEDD and LVESD parameters and higher pulmonary artery pressures. Approximately two-thirds of the cases $(149 / 218,68.4 \%)$ were classified as having a degenerative aetiology for their MR, while the remaining cases had a functional aetiology. The mean ERO area was 0.42(SD: 0.30) $\mathrm{cm}^{2}$. The median time duration of follow-up from the date of initial echocardiography was 31 months (IQR: 12-45 months). The total patient time available was 13028 months.

\section{Clinical outcomes}

Patients with significant MR were more likely to experience hospital admissions for heart failure (table 2). The greatest relative increase in heart failure admissions was evident at 12 months. The time to any rehospitalisation or heart failure rehospitalisation was shorter among the patients with significant MR but the difference was not significant. Overall, significant MR was associated with a higher rate of mortality across the follow-up period, with this difference being evident at 12 months but diminishing over the longer term. The Kaplan-Meier survival plots for the entire cohort and patients over 75 years are presented in figures 2 and 3, respectively. No differences were observed in the rates of stroke or atrial fibrillation admissions.

\section{Multivariate impact of significant MR}

In a multivariate analysis (table 3), increasing age and worsening $\mathrm{LV}$ function were associated with a stepwise increase in the HR for mortality. A similar increase in HR for mortality was seen with a history of chronic obstructive airways disease and an increasing Charlson index. An interaction between a previous ACS event and GFR $<30 \mathrm{~mL} / \mathrm{min} / 1.73 \mathrm{~m}^{2}$ and chronic obstructive airways disease, respectively, was also observed. Within this model, a significant MR was associated with a significant increase in the risk of late mortality (HR: 1.83, 95\% (CI 1.28 to 2.62, $\mathrm{p}=0.001$ ). The win ratio for death during the follow-up period was 0.57 (95\% CI 0.40 to $0.78, \mathrm{p}=0.0002)$, and the win ratio for death or heart failure readmission was $0.53(95 \%$ CI 0.39 to 0.71 , $\mathrm{p}<0.0001)$. Given the two separate aetiologies of MR observed in this study, an interaction analysis was 
Table 2 Clinical outcomes and heart failure admission characteristics with and without significant MR

\begin{tabular}{|c|c|c|c|}
\hline Characteristic & $\begin{array}{l}\text { Without significant MR } \\
\mathrm{N}=218\end{array}$ & $\begin{array}{l}\text { With significant MR } \\
\mathrm{N}=218\end{array}$ & p Value \\
\hline Death (n, \%) & $90(41.3)$ & $115(52.7)$ & 0.021 \\
\hline 30-day death (n, \%) & $15(6.9)$ & $22(10.1)$ & 0.303 \\
\hline 12-month death $(n, \%)$ & $40(18.3)$ & $69(31.7)$ & 0.002 \\
\hline Heart failure admission ( $n, \%)$ & $23(10.5)$ & $41(18.8)$ & 0.021 \\
\hline 30-day HF admission (n, \%) & $4(1.8)$ & $7(3.2)$ & 0.544 \\
\hline 12-month HF admission ( $\mathrm{n}, \%$ ) & $13(6.0)$ & $27(12.4)$ & 0.030 \\
\hline $\mathrm{AF}$ admission $(\mathrm{n}, \%)$ & $9(4.1)$ & $12(5.5)$ & 0.656 \\
\hline Stroke admission (n, \%) & $3(1.3)$ & $0(0)$ & - \\
\hline Median number of admissions ( $n, I Q R)$ & $4(2-7)$ & $4(2-6)$ & 0.554 \\
\hline Median total hospital days (n, IQR) & $23(8-55)$ & $21(9-48)$ & 0.873 \\
\hline Median time to first readmission days (n, IQR) & $77(15-293)$ & $40(14-161)$ & 0.116 \\
\hline Median number of HF admissions (n, IQR) & $1(1)$ & $1(1-2)$ & 0.233 \\
\hline Median total HF hospital days (n, IQR) & $7(5-18)$ & $8(4-18)$ & 0.961 \\
\hline Median time to first HF readmission days ( $n, I Q R)$ & $225(48-799)$ & $135(41-503)$ & 0.162 \\
\hline
\end{tabular}

Values are mean $\pm \mathrm{SD}, \mathrm{n}(\%)$ or median (IQR).

$\mathrm{AF}$, atrial fibrillation; $\mathrm{HF}$, heart failure; MR, mitral regurgitation.

performed to evaluate the differing HRs on survival associated with each respective aetiology. No statistical interaction was observed within this analysis (interaction $\mathrm{p}$ value $=0.108)$, hence indicating that the overall analysis provided the most robust estimate of effect.

\section{Impact of significant MR on survival}

The model was used to explore the reduction in median survival due to significant MR. Among patients with no significant comorbidity and normal LV function, the presence of significant MR was associated with a median survival that was well beyond the 4-year observation period of our data, though a substantial reduction in survival remained evident as compared to patients without significant MR. Similarly, patients with significant MR aged 75 to 85 years with severe LV systolic dysfunction without significant comorbidities had a median survival of 2.67 years compared to similar patients without significant MR who experienced a median survival of 4.96 years. This translates to a reduction of survival by 2.29 years. However, among patients with significant MR, above 85 years of age with severe LV dysfunction, GFR $<30 \mathrm{~mL} / \mathrm{min} / 1.73 \mathrm{~m}^{2}$, a history of coronary artery disease, Charlson index of two or more, the median survival was a meagre 0.07 years. Without significant MR, this survival was only extended to 0.27 years. The reduction of survival attributable to significant MR in this context was only 0.20 years. A prevailing trend of gradual diminishment in the projected median survival between various patient groups was observed. Figure 4 presents the predicted survival and relative impact of significant $\mathrm{MR}$ in patients with various degrees of comorbidities.

\section{DISCUSSION}

Significant MR, especially of the degenerative variant, is an emerging public health problem as a consequence of the population ageing and growth. ${ }^{1} \mathrm{~A}$ current understanding of the natural history of significant MR has been derived from research of select patient cohorts, often excluding complex comorbid patients. ${ }^{16}$ In the absence of clinical trials for the broader realworld population with significant MR, ${ }^{10}$ the current challenge lies therein in devising the optimal management strategy for the ageing, complex comorbid patient with significant MR. As such, quantifying the independent prognostic impact of significant MR in the presence of competing comorbidities becomes even more relevant, as it facilitates clinical decision-making regarding patient management and utilisation of novel interventional therapeutic modalities.

This analysis focuses on defining the independent impact of significant MR on the individual, after accounting for age and comorbidities, within a realworld population of patients undergoing echocardiography. As observed, significant MR was associated with an increase in the risk of death and recurrent hospitalisation for heart failure that was of similar magnitude. Using the 'win-ratio' methodology, it was further demonstrated that patients with significant MR experience these end points at nearly twice the rate of similar patients without significant MR.

Furthermore, best estimation of a robust model demonstrated that significant MR was associated with the most substantial reductions in the projected median survival among patients aged between 65 and 85 years with severe LV dysfunction and limited comorbidities. In contrast, the presence of significant MR in patients with severe LV dysfunction and multiple comorbidities conferred a lesser absolute reduction on the projected median survival, since increasing non-cardiac comorbidities had a substantial impact on overall survival, leading to a relatively small difference in the median survival attributable to significant MR. This finding is especially important, as applying novel percutaneous MR 


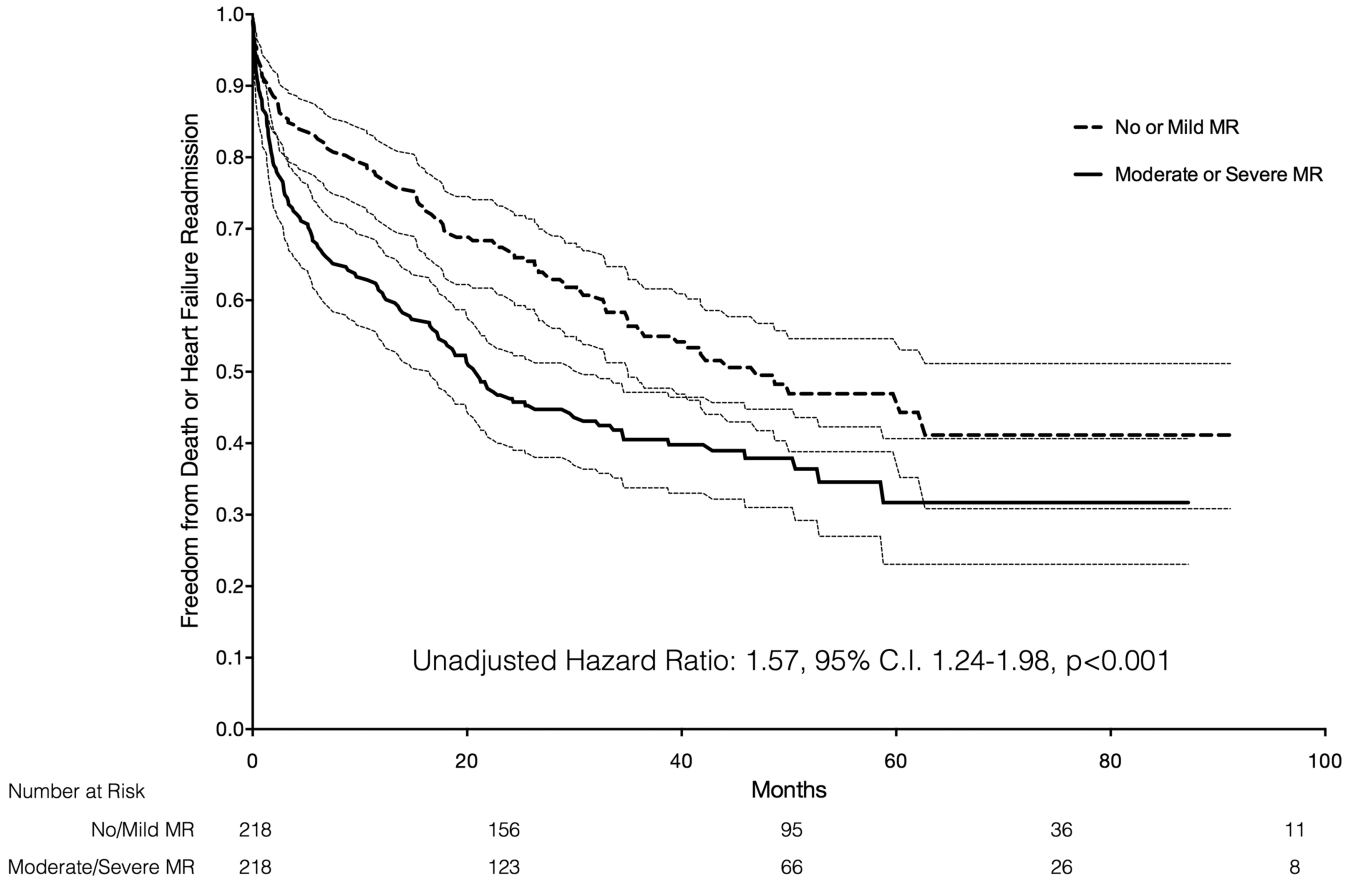

Figure 2 Freedom from death or heart failure readmission over 5 years (unadjusted) between patients with and without significant mitral regurgitation.

corrective technology in such highly comorbid patients with reduced expected survival may be of limited value. While the impact of this novel technology on survival may be diminished in this particular patient cohort, a critical evaluation should also consider its impact on heart failure hospitalisation, improvements in quality of life and functional capacity.
Previous efforts to quantify the mortality risk associated with significant MR have focused on a population of patients with existing heart failure, finding HRs for mortality of 2.0 (95\% CI 1.5 to $2.6, \mathrm{p}<0.001)$ and 1.9 (95\% CI 1.3 to $2.9, \mathrm{p}<0.001)$ for patients with dilated and non-dilated cardiomyopathy, respectively. ${ }^{21}$ As for the population of patients without pre-existing heart

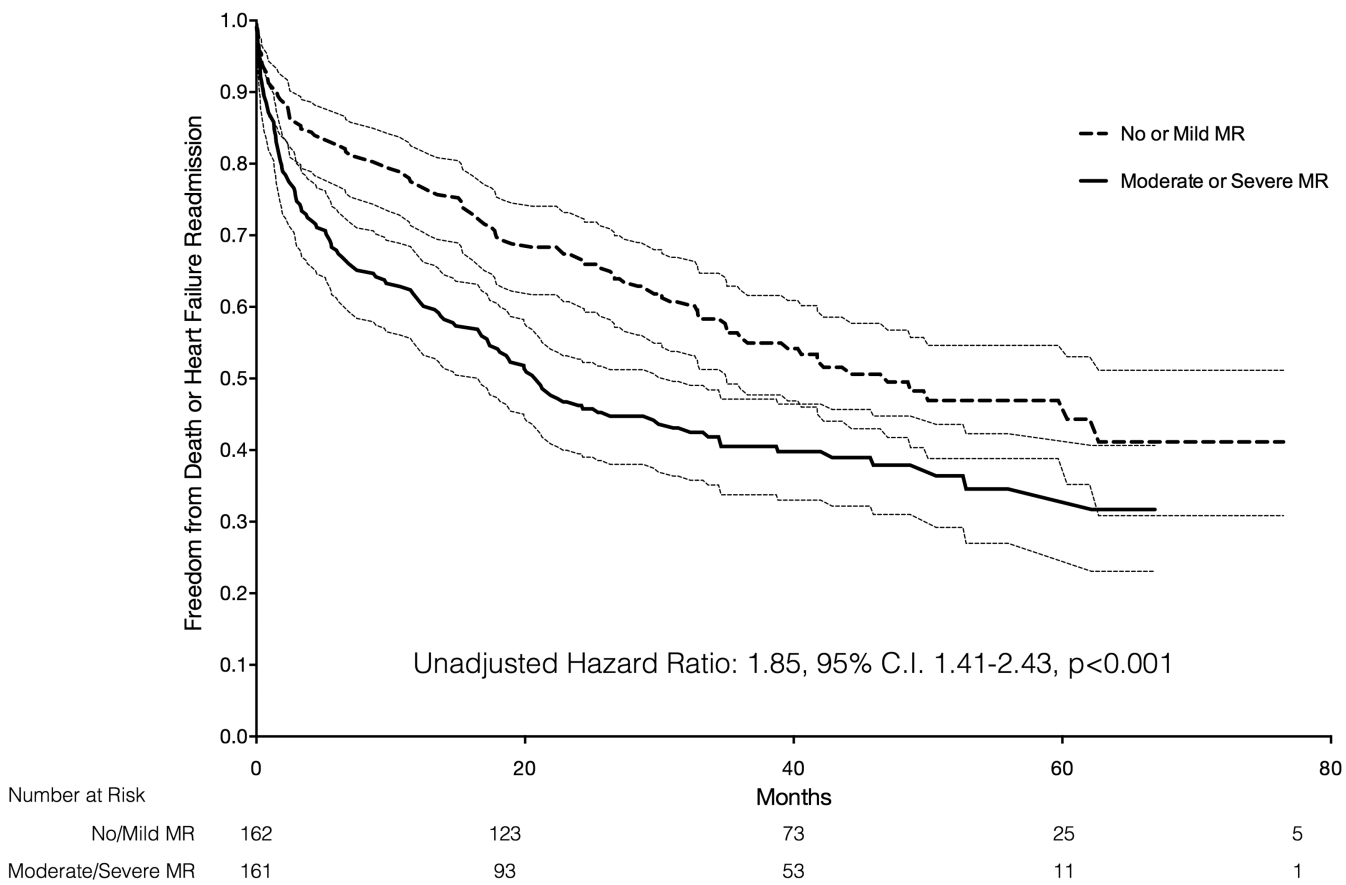

Figure 3 Kaplan-Meier curve for population $>75$ years censored to 5 years. 
Table 3 Cox proportional hazards model predicting survival for patients with significant mitral regurgitation and other clinical comorbidities

\begin{tabular}{|c|c|c|c|}
\hline Characteristic & HR & $95 \% \mathrm{Cl}$ & $\bar{p}$ Value \\
\hline Grade 3 or 4 MR (significant MR) univariate & 1.47 & 1.13 to 1.92 & 0.005 \\
\hline Grade 3 or 4 MR (significant MR) multivariate & 1.83 & 1.28 to 2.62 & $<0.001$ \\
\hline Known lung disease & 2.75 & 1.70 to 4.43 & $<0.001$ \\
\hline MR interaction with known lung disease & 1.98 & 0.44 to 8.82 & 0.005 \\
\hline \multicolumn{4}{|l|}{ Age group } \\
\hline $65-75$ years & 3.52 & 1.47 to 8.42 & 0.005 \\
\hline $75-85$ years & 4.73 & 2.17 to 10.3 & $<0.001$ \\
\hline$>85$ years & 7.01 & 3.24 to 15.2 & $<0.001$ \\
\hline \multicolumn{4}{|l|}{ LV dysfunction } \\
\hline Mild & 0.91 & 0.62 to 1.35 & 0.641 \\
\hline Moderate & 1.13 & 0.77 to 1.65 & 0.546 \\
\hline Severe & 1.75 & 1.19 to 2.58 & 0.004 \\
\hline Previous ACS & 1.00 & 0.67 to 1.51 & 0.99 \\
\hline GFR $<30 \mathrm{~mL} / \mathrm{min} / 1.73 \mathrm{~m}^{2}$ & 1.29 & 0.77 to 2.17 & 0.34 \\
\hline Previous ACS/CAD interaction with GFR $<30 \mathrm{~mL} / \mathrm{min} / 1.73 \mathrm{~m}^{2}$ & 2.97 & 1.47 to 5.93 & 0.019 \\
\hline Previous ACS/CAD interaction with known lung disease & 1.29 & 0.65 to 2.50 & 0.026 \\
\hline Charlson index 1 & 2.05 & 1.26 to 3.32 & 0.004 \\
\hline Charlson index 2 & 2.50 & 1.64 to 3.81 & $<0.001$ \\
\hline
\end{tabular}

failure, a recent analysis of significant MR from the Mitral Regurgitation International Database (MIDA) registry demonstrated mortality rates of 16,31 and $59 \%$ at 5,10 and 20 years, respectively, from diagnosis for those receiving initial medical management. ${ }^{22}$ Within our analysis of the broader population of patients with and without heart failure, we find a consistent degree of excess risk. Interestingly, the overall rates of mortality were higher in this analysis, which may be attributable to the older median age of the population studied.

An additional observation from the Cox model was that of no interaction between reduced LV function and the impact of significant MR; the clinical impact of MR on mortality was evident among patients with normal LV function. In contrast, a significant interaction between significant MR and a history of lung disease was

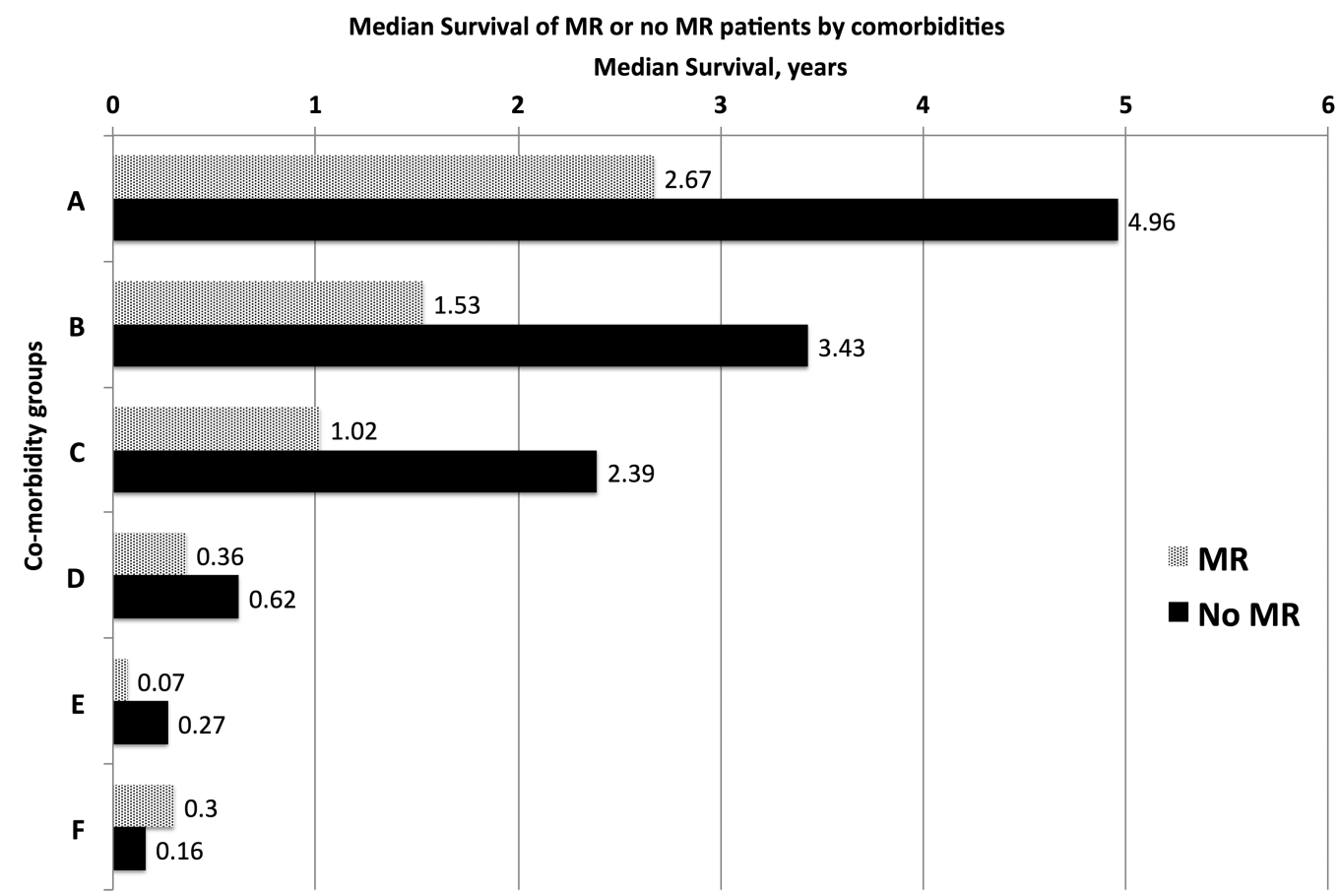

Figure 4 Median survival of patients with and without significant mitral regurgitation according to burden of comorbidities. 
observed. Among patients with existing lung disease, significant MR had little or no effect on median survival. However, survival among patients with severe lung disease is very poor, ${ }^{23}$ and therefore a negative impact of significant MR among these patients is harder to demonstrate.

When considering the application of emerging technologies for the treatment of significant MR, it is anticipated that these will be used among patients with advanced comorbidities, deemed unsuitable for open surgical mitral valve repair or replacement. ${ }^{24} 25$ Our current analysis seeks to help to define the appropriate clinical population that should be studied in future clinical trials for emerging technologies.

This study has demonstrated that the relevance of the MR needs to be evaluated within the context of a greater burden of comorbid disease. Using the multivariate model projections to estimate the reduction in median survival from MR seeks to explore the interface between MR and patient comorbidities. Within this analysis, additional comorbidities, with the exception of lung disease, appear to be associated with an increase in the life lost due to significant MR and therefore the opportunity to benefit from novel interventions. Of course, among patients with multiple comorbidities, survival is also poor, and as a consequence, the impact of significant MR is smaller and the benefit of novel interventions may be more limited. These findings suggest that the opportunity to provide benefit to patients with significant MR through emerging technologies may be of greater value among those patients with impaired $\mathrm{LV}$ function but limited non-cardiac comorbidities.

\section{CONCLUSION}

Across a broad spectrum of patients, significant MR is associated with a substantial increase in the risk of death or heart failure hospitalisation. The presence of significant MR is associated with a marked reduction in median survival, though this difference diminishes in the presence of substantial non-cardiac comorbidities. Such analyses may aid in the selection of patient subgroups that would benefit from emerging technologies targeting significant MR.

\section{Study limitations}

This analysis is observational in nature and therefore is subject to the inherent issues of residual unmeasured biases. Nevertheless, consistency between these findings and previous estimates of risk associated with significant MR among patients with heart failure may suggest that these hidden biases are limited. In addition, the generalisability of the projected estimates of the years of life lost is dependent on the robustness of the model and its relevance to other patient populations with significant MR. The differences of median survival in patients with and without MR in each depicted patient group are only best estimates. Nevertheless, such analyses are best used to explore the intersection between specific conditions and the emerging burden of multiorgan comorbidities that is increasingly prevalent as the population ages. The projected relative impact is of potential utility for informing patient selection in order to improve the design of future pragmatic clinical trials and concurrent health technology evaluations that will be required with the many emerging technologies for the percutaneous treatment of mitral regurgitation.

Contributors RP contributed to the data analysis, drafting and revision of the manuscript. DPC conceived the study question, designed the study, performed the data analysis, drafted the initial manuscript and revised the manuscript for intellectual content. MH contributed to the data collection, organisation of data entry and revision of the final manuscript. AM, MP, LL and AS contributed to the data collection, analysis, echocardiographic analysis and revision of the final manuscript. MXJ contributed to the echocardiographic analysis and revision of the final manuscript.

Funding The authors received funding assistance for this research through a grant from Abbott Vascular, Santa Clara, California, USA

Competing interests None.

Ethics approval This study was approved by the Flinders Medical Centre Human Research Ethics Committee (HREC).

Provenance and peer review Not commissioned; externally peer reviewed.

Data sharing statement No additional data are available.

Open Access This is an Open Access article distributed in accordance with the Creative Commons Attribution Non Commercial (CC BY-NC 3.0) license, which permits others to distribute, remix, adapt, build upon this work noncommercially, and license their derivative works on different terms, provided the original work is properly cited and the use is non-commercial. See: http:// creativecommons.org/licenses/by-nc/3.0/

\section{REFERENCES}

1. Nkomo VT, Gardin JM, Skelton TN, et al. Burden of valvular heart diseases: a population-based study. Lancet 2006;368:1005-11.

2. lung $B$, Baron $G$, Butchart $E G$, et al. A prospective survey of patients with valvular heart disease in Europe: the Euro Heart Survey on valvular heart disease. Eur Heart J 2003;24:1231-4.

3. Enriquez-Sarano M, Avierinos JF, Messika-Zeitoun D, et al. Quantitative determinants of the outcome of asymptomatic mitral regurgitation. N Engl J Med 2005;352:875-83.

4. Grigioni F, Tribouilloy C, Averinos JF, et al. Outcomes in mitral regurgitation due to flail leaflets. J Am Coll Cardiol Img 2008;1:133-41.

5. Ling LH, Enriquez-Sarano M, Seward JB, et al. Clinical outcome of mitral regurgitation due to flail leaflet. $N$ Engl $J$ Med 1996;335:1417-23.

6. Rosen SE, Borer JS, Hochreiter C, et al. Natural history of the asymptomatic/minimally symptomatic patient with severe mitral regurgitation secondary to mitral valve prolapse and normal right and left ventricular performance. Am J Cardiol 1994;74:374-80.

7. Bonow RO, Carabello BA, Chatterjee K, et al. ACC/AHA 2006 Guidelines for the Management of Patients with Valvular Heart Disease : a report of The American College of Cardiology/American Heart Association Task Force on Practice Guidelines (Writing Committee to Revise the 1998 Guidelines for The Management of Patients with Valvular Heart Disease) : developed in collaboration with the Society of Cardiovascular Anaesthesiologist : endorsed by the Society for Cardiovascular Angiography and Interventions and the Society of Thoracic Surgeons. Circulation 2006;114:e84-231.

8. Vahanian A, Alfieri O, Andreotti F, et al. Guidelines on the management of valvular heart disease (version 2012). The Joint Task Force on the Management of Valvular Heart Disease of the European Society of Cardiology (ESC) and the European Association for Cardio-Thoracic Surgery (EACTS). Eur Heart $J$ 2012;33:2451-96.

9. Zoghbi WA, Enriquez-Sarano M, Foster E, et al. Evaluation of the severity of native valvular regurgitation with two-dimensional and Doppler echocardiography: a report from the Task Force on Valvular 
Regurgitation of the American Society of Echocardiography. Am Soc Echocardiogr 2003;16:777-802.

10. Enriquez-Sarano M, Akins CW, Vahanian A. Mitral regurgitation Lancet 2009;373:1382-94.

11. Hochreiter C, Niles N, Devereux RB, et al. Mitral regurgitation: relationship of noninvasive descriptors of right and left ventricular performance to clinical and hemodynamic findings and to prognosis in medically and surgically treated patients. Circulation 1986;73:900-12.

12. Ramanathan KB, Knowles J, Connor MJ, et al. Natural history of chronic mitral insufficiency: relation of peak systolic pressure/ end-systolic volume ration to morbidity and mortality. J Am Coll Cardiol 1984;3:1412-16.

13. DePace NL, Mintz GS, Ren JF, et al. Natural history of the flail mitra leaflet syndrome: serial 2-dimensional echocardiographic study. Am J Cardiol 1983;52:789-95.

14. Hammermeister KE, Fisher L, Kennedy W, et al. Prediction of late survival in patients with mitral valve disease from clinical, hemodynamic and quantitative angiographic variables. Circulation 1978;57:341-9.

15. Delahaye JP, Gare JP, Viguier E, et al. Natural history of severe mitral regurgitation. Eur Heart J 1991;12(Suppl B):5-9.

16. lung $B$, Vahanian $A$. Epidemiology of valvular heart disease in the adult. Nat Rev Cardiol 2011;8:162-72.

17. Charlson ME, Pompei $\mathrm{P}$, Ales $\mathrm{KL}$, et al. A new method of classifying prognostic comorbidity in longitudinal studies: development and validation. J Chronic Dis 1987;40:373-83.

18. Quinones MA, Douglas PS, Foster E, et al. ACC/AHA clinical competence statement on echocardiography: a Report of the
American College of Cardiology/American Heart Association American College of Physicians-American Society of Internal Medicine Task Force on Clinical Competence Developed in Collaboration with the American Society of Echocardiography, the Society of Cardiovascular Anaesthesiologist and the Society of Paediatric Echocardiography. J Am Coll Cardiol 2003; 41:687-708.

19. Pocock SJ, Ariti CA, Collier TJ, et al. The win ratio: a new approach to the analysis of composite endpoints in clinical trials based on clinical priorities. Eur Heart J 2012;33:176-82.

20. Finkelstein DM, Schoenfeld DA. Combining mortality and longitudinal measures in clinical trials. Stat Med 1999; 18:1341-54.

21. Rossi A, Dini FL, Faggiano $P$, et al. Independent prognostic value of functional mitral regurgitation in patients with heart failure. A quantitative analysis of 1256 patients with ischaemic and non-ischaemic dilated cardiomyopathy. Heart 2011;97:1675-80.

22. Suri RM, Vanoverschelde JL, Grigioni F, et al. Association between early surgical intervention vs watchful waiting and outcomes for mitral regurgitation due to flail mitral leaflets. JAMA 2013;310:609-16.

23. Centers for Disease Control and Prevention (CDC). Chronic obstructive pulmonary disease among adults-United States, 2011. MMWR Morb Mortal Wkly Rep 2012;61:938.

24. Feldman T, Foster E, Glower DG, et al. Percutaneous repair or surgery for mitral regurgitation. N Engl J Med 2011;364:1395-406.

25. Whitlow PL, Feldman T, Pedersen WR, et al. Acute and 12-month results with catheter-based mitral valve leaflet repair. J Am Coll Cardiol 2012;59:130-9. 